\title{
Selfadjoint extensions of the multiplication operator in de Branges spaces as singular rank-one perturbations
}

\author{
Luis O. Silva* \\ Departamento de Física Matemática \\ Instituto de Investigaciones en Matemáticas Aplicadas y en Sistemas \\ Universidad Nacional Autónoma de México \\ C.P. 04510, México D.F. \\ silva@iimas. unam.mx \\ Julio H. Toloza ${ }^{\dagger}$ \\ Instituto de Matemática de Bahía Blanca \\ Universidad Nacional del Sur \\ Consejo Nacional de Investigaciones Científicas y Técnicas \\ Departamento de Matemática \\ Av. Alem 1253, B8000CPB Bahía Blanca, Argentina \\ julio.toloza@uns. edu. ar
}

\begin{abstract}
We derive a description of the family of canonical selfadjoint extensions of the operator of multiplication in a de Branges space in terms of singular rank-one perturbations using distinguished elements from the set of functions associated with a de Branges space. The scale of rigged Hilbert spaces associated with this construction is also studied from the viewpoint of de Branges's theory.
\end{abstract}

Mathematics Subject Classification(2010): Primary 46E22, 47A70; Secondary 47A55, 47B15, 47B25.

Keywords: de Branges spaces; singular rank-one perturbations; scale of Hilbert spaces.

*Partially supported by SEP-CONACYT (Mexico) under Grant CB-2015 254062

${ }^{\dagger}$ Partially supported by CONICET (Argentina) under Grant PIP 11220150100327CO 


\section{Introduction}

In de Branges's theory of Hilbert spaces of entire functions, the operator of multiplication by the independent variable plays a central role. From particular features of this operator, one can infer properties of concrete realizations of de Branges spaces. Conversely, the particularities of a de Branges space determine the spectral properties of the selfadjoint extensions of the corresponding multiplication operator. This fact is specially useful when, via the so-called functional model, one can identify Krein's entire operators and, more generally, $n$-entire operators (in particular, regular and singular Schrödinger operators as well as Jacobi operators) with the multiplication operator in certain de Branges spaces [14-16, 18-21].

This article uses singular perturbation theory for dealing with the family of canonical selfadjoint extensions $S_{\gamma}$ of the multiplication operator $S$ in a de Branges space $\mathcal{B}$. Our approach to this issue is not reduced to the application of perturbation theory to a concrete family of operators. Instead, we focus our attention to functions in de Branges space theory that play a central role when considering the operators $S_{\gamma}$ as a family of singular rank-one perturbations of a certain selfadjoint extension of $S$. To the best of our knowledge, this way of dealing with the matter is new. We restrict our considerations to the case when the operator $S$ is densely defined since the other case has already been treated in [22]. As a matter of fact, this work can be regarded as a further development of the results given in that paper. Singular perturbations are treated by means of triplets of Hilbert spaces [4, Chapter I]. We combine this operator-theoretic approach with the properties of functions in both the de Branges space and its set of associated functions assoc $\mathcal{B}$ (see Section 2). In doing so, we have two goals in mind. The first one consists in shedding light on the properties of the linear spaces involved in the theory of triplets of Hilbert spaces. The second aim concerns the incorporation of de Branges's theory into the theoretical framework of singular rank-one perturbations. The results concerning the first goal can be summarized as follows: Let $\mathcal{B}_{+2}^{(\gamma)}$ and $\mathcal{F}_{+1}$ be $\operatorname{dom}\left(S_{\gamma}\right)$ and $\operatorname{dom}\left(S^{*}\right)$ with their respective graph norms. Let $\mathcal{B}_{-2}^{(\gamma)}$ and $\mathcal{F}_{-1}$ be their respective duals. By standard theory, one has

$$
\mathcal{B}_{+2}^{(\gamma)} \subset \mathcal{F}_{+1} \subset \mathcal{B} \subset \mathcal{F}_{-1} \subset \mathcal{B}_{-2}^{(\gamma)}
$$

We prove that $\mathcal{B}_{+2}^{(\gamma)}$ is a de Branges space (Theorem 3.1) and the space $\mathcal{F}_{+1}$ share many of the properties of a de Branges space (Theorem 3.2) but not all of them (Example 3.3). We next prove that $\mathcal{F}_{-1}$ is realized by (that is, is 
isometrically isomorphic to) a de Branges space which is setwise equal to assoc $\mathcal{B}$ (Proposition 3.4 and Theorem 3.5); we remark that $\mathcal{F}_{-1}$ is initially a Hilbert space of continuous linear functionals acting on (the Hilbert space of entire functions) $\mathcal{F}_{+1}$. Finally, we show that $\mathcal{B}_{-2}^{(\gamma)}$ is not a de Branges space but rather a quotient space involving assoc $\mathcal{B}$ (Theorem 3.7).

On the subject of the second goal, our approach allows us to find formulae for rendering the family of selfadjoint extensions of the multiplication operator as a family of rank-one singular perturbations. Namely (Theorems 4.7 and 4.9),

$$
\operatorname{dom}\left(S_{\gamma}\right)=\left\{\begin{array}{c}
g(z)=h(z)+b S_{\pi / 2}\left(S_{\pi / 2}+i I\right)^{-1} k(z,-i) \\
h(z) \in \operatorname{dom}\left(S_{\pi / 2}\right), b \in \mathbb{C}:\left\langle s_{0}, h\right\rangle_{2}=\pi b\left(\tan \gamma+\operatorname{re} \frac{s_{0}(i)}{s_{\pi / 2}(i)}\right)
\end{array}\right\}
$$

where $k(z, w)$ is the reproducing kernel in $\mathcal{B}$ and the functions $s_{\gamma}(z)$ are given in (2). Then the operator $S_{\gamma}$ is the restriction of

$$
\tilde{S}_{\gamma}:=\tilde{S}_{\pi / 2}-\frac{\cot \gamma}{\pi}\left\langle s_{0}, \cdot\right\rangle_{\mathcal{F}} s_{0}(z)
$$

to $\operatorname{dom}\left(S_{\gamma}\right)$; we note that $\tilde{S}_{\gamma}$ are maps from $\operatorname{dom}\left(S^{*}\right)$ to $\mathcal{B}_{-2}^{(\pi / 2)}$ (the details are discussed in Section 4). Although, in an abstract setting, this kind of formulae are known, here they are derived using function theoretical methods pertaining to de Branges theory that make clear the prominent role played by the functions $s_{\gamma}(z)$ in these formulae. Furthermore, we obtain as by-products the Krein's formula for resolvents and some objects related to it in terms of functions in de Branges's theory.

\section{Remarks on de Branges Hilbert spaces}

Throughout this paper, inner products in Hilbert spaces are assumed conjugate linear with respect to the left argument. We follow the customary rule of denoting a function $f$ by its evaluation at an arbitrary value of its argument $f(z)$. Also, we often denote the action of an operator $B$ on a function $f(z)$ by $B f(z)$ instead of $(B f)(z)$.

A Hilbert space of entire functions $\mathcal{B}$ is a de Branges $(\mathrm{dB})$ space if it has a reproducing kernel and is isometrically invariant under both the conjugation 
$f(z) \mapsto f^{\#}(z):=\overline{f(\bar{z})}$ and the mapping

$$
f(z) \mapsto \frac{z-\bar{w}}{z-w} f(z)
$$

whenever $w \in \mathbb{C}$ is a non real zero of $f(z)$.

Alternatively, dB spaces can be defined in terms of functions of the HermiteBiehler class $\mathcal{H B}$, that is, entire functions for which the inequality $|e(z)|>|e(\bar{z})|$ holds for all $z \in \mathbb{C}_{+}$. Indeed, given $e(z) \in \mathcal{H B}$, one defines

$$
\mathcal{B}(e):=\left\{f(z) \text { entire }: \frac{f(z)}{e(z)}, \frac{f^{\#}(z)}{e(z)} \in \mathcal{H}^{2}\left(\mathbb{C}_{+}\right)\right\}
$$

where $\mathcal{H}^{2}\left(\mathbb{C}_{+}\right)$is the Hardy space on the upper half plane; the inner product in $\mathcal{B}(e)$ is given by

$$
\langle f, g\rangle:=\int_{\mathbb{R}} \frac{\overline{f(x)} g(x)}{|e(x)|^{2}} d x .
$$

According to [7, Problem 50] $\mathcal{B}(e)$ so defined is a $\mathrm{dB}$ space with reproducing kernel

$$
k(z, w)= \begin{cases}\frac{e^{\#}(z) e(\bar{w})-e(z) e^{\#}(\bar{w})}{2 \pi i(z-\bar{w})}, & w \neq \bar{z}, \\ \frac{e^{\#^{\prime}}(z) e(z)-e^{\prime}(z) e^{\#}(z)}{2 \pi i}, & w=\bar{z} .\end{cases}
$$

On the other hand, given a $\mathrm{dB}$ space $\mathcal{B} \neq\{0\}$ there exists $e(z) \in \mathcal{H} \mathcal{B}$ such that $\mathcal{B}=\mathcal{B}(e)$ isometrically [7, Theorem 23]; such a function however is not unique [6, Theorem 1].

A entire function $h(z)$ is associated to a given $\mathrm{dB}$ space $\mathcal{B}$ if

$$
\frac{h(w) f(z)-h(z) f(w)}{z-w} \in \mathcal{B}
$$

for every $f(z) \in \mathcal{B}$ and $w \in \mathbb{C}$ such that $h(w) \neq 0$. The set of associated functions is denoted assoc $\mathcal{B}$. By $[13$, Lemma 4.5] one has assoc $\mathcal{B}=z \mathcal{B}+\mathcal{B}$. Within assoc $\mathcal{B}$ lies the family of functions

$$
s_{\gamma}(z):=\frac{i}{2}\left[e^{i \gamma} e(z)-e^{-i \gamma} e^{\#}(z)\right], \quad \gamma \in[0, \pi)
$$

These functions are in bijective correspondence with the family of canonical selfadjoint extension of the multiplication operator; see below. We note that, 
in general, $s_{\gamma}(z) \in \operatorname{assoc} \mathcal{B} \backslash \mathcal{B}$ with the possible exception of at most one of such functions [5, Lemma 7]. Extending the definition (2) to $\gamma \in \mathbb{R}$ one has the identity

$$
s_{\gamma}(z)=\cos \left(\gamma-\gamma_{0}\right) s_{\gamma_{0}}(z)+\sin \left(\gamma-\gamma_{0}\right) s_{\gamma_{0}+\pi / 2}(z),
$$

where $\gamma_{0}$ is fixed but otherwise arbitrary. Also,

$$
k(z, w)= \begin{cases}\frac{s_{\gamma_{0}+\pi / 2}(z) s_{\gamma_{0}}(\bar{w})-s_{\gamma_{0}+\pi / 2}(\bar{w}) s_{\gamma_{0}}(z)}{\pi(z-\bar{w})}, & z \neq \bar{w} \\ \frac{s_{\gamma_{0}+\pi / 2}^{\prime}(z) s_{\gamma_{0}}(z)-s_{\gamma_{0}+\pi / 2}(z) s_{\gamma_{0}}^{\prime}(z)}{\pi}, & z=\bar{w} .\end{cases}
$$

The operator of multiplication by the independent variable is defined by $(S f)(z)=z f(z)$ with domain $\operatorname{dom}(S)$ maximal in $\mathcal{B}$. This operator is closed, completely nonselfadjoint and has deficiency indices $(1,1)$. Additionally, $S$ is also regular, i. e. its spectral core is empty, if and only if $\mathcal{B}=\mathcal{B}(e)$ with $e(z) \in$ $\mathcal{H B}$ devoid of zeros in the real line. Also, $\operatorname{dom}(S)$ may be either dense in $\mathcal{B}$ or has codimension equal to one; the latter happens if and only if there exists (a necessarily unique) $\gamma \in[0, \pi)$ such that $s_{\gamma}(z) \in \mathcal{B}$, in which case $s_{\gamma}(z)$ is orthogonal to $\operatorname{dom}(S)[7]$.

From this point on, we consider only dB spaces with the property of $S$ being densely defined; dB spaces with $\operatorname{dom}(S)$ having non zero codimension have been discussed in [22].

Observe that

$$
\left\langle\left(S^{*}-\bar{w}\right) k(\cdot, w), g(\cdot)\right\rangle=\langle k(\cdot, w),(S-w) g(\cdot)\rangle=0
$$

for all $g(z) \in \operatorname{dom}(S)$. Therefore

$$
k(z, w) \in \operatorname{ker}\left(S^{*}-\bar{w} I\right), \quad w \in \mathbb{C} .
$$

Thus, since we assume $S$ densely defined, $S^{*}$ can be described as

$$
\begin{gathered}
\operatorname{dom}\left(S^{*}\right)=\left\{\begin{array}{c}
g(z)=h(z)+a^{(+)} k(z, \bar{w})+a^{(-)} k(z, w): \\
h(z) \in \operatorname{dom}(S), a^{( \pm)} \in \mathbb{C}, \operatorname{im}(w)>0
\end{array}\right\}, \\
S^{*} g(z)=z h(z)+w a^{(+)} k(z, \bar{w})+\bar{w} a^{(-)} k(z, w) .
\end{gathered}
$$

The canonical selfadjoint extensions of $S$ are the selfadjoint restrictions of 
$S^{*}$. A standard description of them is given in [13], where the connection to the family of functions $s_{\gamma}(z)$ is made explicit:

$$
\begin{gathered}
\operatorname{dom}\left(S_{\gamma}\right)=\left\{g(z)=\frac{f(z)-\frac{s_{\gamma}(z)}{s_{\gamma}(w)} f(w)}{z-w}: f(z) \in \mathcal{B}, w \in \mathbb{C} \backslash \mathbb{R}\right\}, \\
S_{\gamma} g(z)=z g(z)+f(w) \frac{s_{\gamma}(z)}{s_{\gamma}(w)}, \quad \gamma \in[0, \pi) .
\end{gathered}
$$

It follows from $(6)$ that $\operatorname{spec}\left(S_{\gamma}\right)=\left\{\right.$ zeros of $\left.s_{\gamma}(z)\right\}$ and the corresponding eigenfunctions are of the form $s_{\gamma}(z) /(z-\lambda), \lambda \in \operatorname{spec}\left(S_{\gamma}\right)$. Note that (6) also implies

$$
\left(S_{\gamma}-w I\right)^{-1} f(z)=g(z), \quad w \notin \operatorname{spec}\left(S_{\gamma}\right) .
$$

If $S$ is also regular, then $\bigcap_{\gamma} \operatorname{spec}\left(S_{\gamma}\right)=\emptyset, \bigcup_{\gamma} \operatorname{spec}\left(S_{\gamma}\right)=\mathbb{R}$, and $\operatorname{spec}\left(S_{\gamma}\right)$ and $\operatorname{spec}\left(S_{\gamma^{\prime}}\right)$ interlace whenever $\gamma \neq \gamma^{\prime}$.

Lemma 2.1. Suppose $v \notin \operatorname{spec}\left(S_{\gamma}\right), w \notin \operatorname{spec}\left(S_{\gamma}\right)$ and $v \neq w$. Then,

$$
\frac{k(z, \bar{w})-k(z, \bar{v})}{w-v}=\left(S_{\gamma}-v I\right)^{-1} k(z, \bar{w})+\frac{1}{w-v}\left(\frac{s_{\gamma}(w)}{s_{\gamma}(v)}-1\right) k(z, \bar{v}) .
$$

Proof. For every $g(z) \in \mathcal{B}$ one has

$$
\begin{aligned}
\left\langle k(\cdot, \bar{w}),\left(S_{\gamma}-\bar{v} I\right)^{-1} g(\cdot)\right\rangle & =\frac{g(\bar{w})-\frac{s_{\gamma}(\bar{w})}{s_{\gamma}(\bar{v})} g(\bar{v})}{\bar{w}-\bar{v}} \\
& =\frac{s_{\gamma}(\bar{w})}{s_{\gamma}(\bar{v})} \frac{g(\bar{v})-\frac{s_{\gamma}(\bar{v})}{s_{\gamma}(\bar{w})} g(\bar{w})}{\bar{v}-\bar{w}} \\
& =\frac{s_{\gamma}(\bar{w})}{s_{\gamma}(\bar{v})}\left\langle k(\cdot, \bar{v}),\left(S_{\gamma}-\bar{w} I\right)^{-1} g(\cdot)\right\rangle
\end{aligned}
$$

SO

$$
s_{\gamma}(v)\left(S_{\gamma}-v I\right)^{-1} k(z, \bar{w})=s_{\gamma}(w)\left(S_{\gamma}-w I\right)^{-1} k(z, \bar{v}) .
$$

Then

$$
\begin{aligned}
k(z, \bar{w})-k(z, \bar{v}) & =\left[\frac{s_{\gamma}(w)}{s_{\gamma}(v)}\left(S_{\gamma}-v I\right)\left(S_{\gamma}-w I\right)^{-1}-I\right] k(z, \bar{v}) \\
& =\left[\left(\frac{s_{\gamma}(w)}{s_{\gamma}(v)}-1\right) I+(w-v) \frac{s_{\gamma}(w)}{s_{\gamma}(v)}\left(S_{\gamma}-w I\right)^{-1}\right] k(z, \bar{v}),
\end{aligned}
$$


yielding the desired result after one more use of (7).

Another consequence of (7) is

$$
s_{\gamma}(\bar{w}) k(z, \bar{w})=s_{\gamma}(w) U(w) k(z, w)
$$

where

$$
U(w):=\left(S_{\gamma}-\bar{w} I\right)\left(S_{\gamma}-w I\right)^{-1}=I+(w-\bar{w})\left(S_{\gamma}-w I\right)^{-1}, \quad w \in \mathbb{C} \backslash \mathbb{R},
$$

is the Cayley transform. This in turn yields another characterization of the adjoint operator.

Lemma 2.2. Given $\gamma \in[0, \pi)$ and $w \in \mathbb{C}: \operatorname{im}(w)>0$, the adjoint operator $S^{*}$ can be described as follows:

$$
\begin{gathered}
\operatorname{dom}\left(S^{*}\right)=\left\{\begin{array}{c}
f(z)=g(z)+b\left(S_{\gamma}-(\operatorname{re} w) I\right)\left(S_{\gamma}-w I\right)^{-1} k(z, w) \\
g(z) \in \operatorname{dom}\left(S_{\gamma}\right), b \in \mathbb{C}
\end{array}\right\}, \\
S^{*} f(z)=S_{\gamma} g(z)+b\left((\operatorname{re} w) S_{\gamma}-|w|^{2} I\right)\left(S_{\gamma}-w I\right)^{-1} k(z, w) .
\end{gathered}
$$

Proof. Define $l(z, w):=k(z, w) / s_{\gamma}(\bar{w})$. Every $f(z) \in \operatorname{dom}\left(S^{*}\right)$ is of the form

$$
f(z)=h(z)+a^{(+)} l(z, \bar{w})+a^{(-)} l(z, w), \quad h(z) \in \operatorname{dom}(S), \quad a^{( \pm)} \in \mathbb{C} .
$$

With the help of (8), this can be written as

$$
f(z)=g(z)+\frac{a^{(+)}+a^{(-)}}{2}(U(w)+I) l(z, w)
$$

where

$$
g(z)=h(z)+\frac{a^{(+)}-a^{(-)}}{2}(U(w)-I) l(z, w)
$$

belongs to the domain of $S_{\gamma}$ (see $\left.(9)\right)$. Since, $U(w)+I=2\left(S_{\gamma}-(\right.$ re $\left.w) I\right)$, which can be verified by a straightforward computation, (10a) has been established.

To prove (10b), it suffices to note that

$$
\begin{aligned}
S^{*} f(z) & =S^{*} g(z)+\frac{a^{(+)}+a^{(-)}}{2}\left(S^{*} l(z, \bar{w})+S^{*} l(z, w)\right) \\
& =S_{\gamma} g(z)+\frac{a^{(+)}+a^{(-)}}{2}(w U(w)+\bar{w} I) l(z, w)
\end{aligned}
$$


and rewrite $w U(w)+\bar{w} I$ using $(9)$.

The description (10) of $S^{*}$ takes its simplest form when $w=i($ or $w=-i)$. In such a case, one obtains (cf. [3])

$$
\begin{gathered}
\operatorname{dom}\left(S^{*}\right)=\left\{\begin{array}{c}
f(z)=g(z)+b S_{\gamma}\left(S_{\gamma}-i I\right)^{-1} k(z, i): \\
g(z) \in \operatorname{dom}\left(S_{\gamma}\right), b \in \mathbb{C}
\end{array}\right\}, \\
S^{*} f(z)=S_{\gamma} g(z)-b\left(S_{\gamma}-i I\right)^{-1} k(z, i) .
\end{gathered}
$$

Remark 1. From this point on, de Branges spaces are assumed to be generated by Hermite-Biehler functions free of zeros on the real line. Equivalently, de Branges spaces are supposed to have the property that, for every $x \in \mathbb{R}$, there exists $f(z)$ in the space such that $f(x) \neq 0$. This assumption, which means that the operator of multiplication is regular, entails no essential loss of generality.

\section{Scales of $\mathrm{dB}$ spaces}

Most of the following discussion is based on [1-3] with some slight modifications. Other related references are [10-12] and the classical book by Berezanskiu [4, Chapter I].

Given $\gamma \in[0, \pi)$, let $\mathcal{B}_{+2}^{(\gamma)}:=\operatorname{dom}\left(S_{\gamma}\right)$ equipped with the graph inner product

$$
\langle f, g\rangle_{+2}:=\left\langle S_{\gamma} f, S_{\gamma} g\right\rangle+\langle f, g\rangle=\left\langle\left(S_{\gamma}-i I\right) f,\left(S_{\gamma}-i I\right) g\right\rangle .
$$

Due to $S_{\gamma}$ being closed, $\mathcal{B}_{+2}^{(\gamma)}$ is a Hilbert space. Its dual $\mathcal{B}_{-2}^{(\gamma)}$ is the completion of $\mathcal{B}$ under the norm induced by the inner product

$$
\langle f, g\rangle_{-2}:=\left\langle f,\left(S_{\gamma}^{2}+I\right)^{-1} g\right\rangle=\left\langle\left(S_{\gamma}-i I\right)^{-1} f,\left(S_{\gamma}-i I\right)^{-1} g\right\rangle .
$$

Since

$$
\left\|S_{\gamma} f\right\|_{-2} \leq\|f\|, \quad f(z) \in \operatorname{dom}\left(S_{\gamma}\right)
$$

the operator $S_{\gamma}$ has a unique contractive continuation $\hat{S}_{\gamma}: \mathcal{B} \rightarrow \mathcal{B}_{-2}^{(\gamma)}$. Similarly,

$$
R_{\gamma}(w):=\left(S_{\gamma}-w I\right)^{-1}: \mathcal{B} \rightarrow \mathcal{B}_{+2}^{(\gamma)}, \quad w \in \mathbb{C} \backslash \operatorname{spec}\left(S_{\gamma}\right)
$$

has a unique bounded extension $\hat{R}_{\gamma}(w): \mathcal{B}_{-2}^{(\gamma)} \rightarrow \mathcal{B}$. The latter operators satisfy 
the resolvent identity

$$
\hat{R}_{\gamma}(w)-\hat{R}_{\gamma}(v)=(w-v) R_{\gamma}(w) \hat{R}_{\gamma}(v)
$$

for $w \in \mathbb{C} \backslash \operatorname{spec}\left(S_{\gamma}\right)$ and $v \in \mathbb{C} \backslash \operatorname{spec}\left(S_{\gamma}\right)$. In terms of $\hat{R}_{\gamma}(w)$, the associated pairing $\langle\cdot, \cdot\rangle_{2}: \mathcal{B}_{-2}^{(\gamma)} \times \mathcal{B}_{+2}^{(\gamma)} \rightarrow \mathbb{C}$ can be written as

$$
\langle\varphi, g\rangle_{2}=\left\langle\hat{R}_{\gamma}(-i) \varphi,\left(S_{\gamma}-i I\right) g\right\rangle, \quad \varphi \in \mathcal{B}_{-2}^{(\gamma)}, \quad g(z) \in \mathcal{B}_{+2}^{(\gamma)} .
$$

Before going into our first result, let us note that

$$
\|g\|_{+2}=\left\|\left(S_{\gamma}-i I\right) g\right\|=\|f\|,
$$

when $g(z)$ and $f(z)$ are related as in $(6 a)$.

Theorem 3.1. $\mathcal{B}_{+2}^{(\gamma)}$ is a dB space.

Proof. The linear functional of point evaluation is continuous in $\mathcal{B}_{+2}^{(\gamma)}$. Indeed, given $g(z) \in \mathcal{B}_{+2}^{(\gamma)}$ there exists $f(z) \in \mathcal{B}$ such that

$$
g(z)=\left(S_{\gamma}-i I\right)^{-1} f(z)
$$

Let $k(w, z)$ be the reproducing kernel in $\mathcal{B}$. Then,

$$
g(z)=\langle k(\cdot, z), g(\cdot)\rangle=\left\langle k(\cdot, z),\left(S_{\gamma}-i I\right)^{-1} f(\cdot)\right\rangle=\left\langle\left(S_{\gamma}+i I\right)^{-1} k(\cdot, z), f(\cdot)\right\rangle
$$

SO

$$
|g(z)| \leq\left\|\left(S_{\gamma}+i I\right)^{-1}\right\|\|k(\cdot, z)\|\|f\|
$$

which along with (14) imply the assertion. Moreover, it follows that

$$
|g(z)-h(z)|^{2} \leq C k(z, z)\|g-h\|_{+2}^{2}
$$

so point evaluation is uniformly continuous over compact subsets of $\mathbb{C}$.

Next, let us verify that the conjugation ${ }^{\#}$ is an isometry in $\mathcal{B}_{+2}^{(\gamma)}$. Consider

$$
g(z)=\left(S_{\gamma}+i I\right)^{-1} f(z)=\frac{f(z)-\frac{s_{\gamma}(z)}{s_{\gamma}(-i)} f(-i)}{z+i}
$$


with $f(z) \in \mathcal{B}$. We have

$$
g^{\#}(z)=\frac{f^{\#}(z)-\frac{s_{\gamma}(z)}{s_{\gamma}(-i)} \overline{f(-i)}}{z-i}=\frac{f^{\#}(z)-\frac{s_{\gamma}(z)}{s_{\gamma}(i)} f^{\#}(i)}{z-i}=\left(S_{\gamma}-i I\right)^{-1} f^{\#}(z),
$$

thus $g^{\#}(z) \in \mathcal{B}_{+2}^{(\gamma)}$ since $f^{\#}(z) \in \mathcal{B}$. Moreover,

$$
\begin{aligned}
\left\|g^{\#}\right\|_{+2}=\left\|\left(S_{\gamma}-i I\right) g^{\#}\right\| & =\left\|f^{\#}\right\| \\
& =\|f\|=\left\|\left(S_{\gamma}+i I\right) g\right\|=\|g\|_{+2},
\end{aligned}
$$

proving the assertion; the fact that the Cayley transform is unitary has been used along the way.

Now, suppose that $v_{0}$ is a non-real zero of $g(z) \in \mathcal{B}_{+2}^{(\gamma)}$ and consider $f(z) \in \mathcal{B}$ such that

$$
g(z)=\left(S_{\gamma}-\overline{v_{0}} I\right)^{-1} f(z)=\frac{f(z)-\frac{s_{\gamma}(z)}{s_{\gamma}\left(\overline{v_{0}}\right)} f\left(\overline{v_{0}}\right)}{z-\overline{v_{0}}} .
$$

Thus, the assumption $g\left(v_{0}\right)=0$ yields

$$
f\left(v_{0}\right)=\frac{s_{\gamma}\left(v_{0}\right)}{s_{\gamma}\left(\overline{v_{0}}\right)} f\left(\overline{v_{0}}\right) .
$$

Hence

$$
\frac{z-\overline{v_{0}}}{z-v_{0}} g(z)=\frac{f(z)-\frac{s_{\gamma}(z)}{s_{\gamma}\left(\overline{v_{0}}\right)} f\left(\overline{v_{0}}\right)}{z-v_{0}}=\frac{f(z)-\frac{s_{\gamma}(z)}{s_{\gamma}\left(v_{0}\right)} f\left(v_{0}\right)}{z-v_{0}}=\left(S_{\gamma}-v_{0} I\right)^{-1} f(z)
$$

so $\left(z-\overline{v_{0}}\right)\left(z-v_{0}\right)^{-1} g(z) \in \mathcal{B}_{+2}^{(\gamma)}$. Finally,

$$
\begin{aligned}
& \left\|\frac{(\cdot)-\overline{v_{0}}}{(\cdot)-v_{0}} g(\cdot)\right\|_{+2}=\left\|\left(S_{\gamma}+i I\right)\left(S_{\gamma}-\overline{v_{0}} I\right)^{-1}\left(S_{\gamma}-\overline{v_{0}} I\right)\left(S_{\gamma}-v_{0} I\right)^{-1} f\right\| \\
& =\left\|\left(S_{\gamma}+i I\right)\left(S_{\gamma}-\overline{v_{0}} I\right)^{-1} f\right\|=\|g\|_{+2},
\end{aligned}
$$

where again the isometric character of the Cayley transform has been used. The proof is now complete. 
Remark 2. Denote by $k_{+2}(z, w)$ the reproducing kernel in $\mathcal{B}_{+2}^{(\gamma)}$. Since

$$
\begin{aligned}
g(w) & =\langle k(\cdot, w), g(\cdot)\rangle \\
& =\left\langle\left(S_{\gamma}+i I\right)\left(S_{\gamma}-i I\right)^{-1} k(\cdot, w),\left(S_{\gamma}+i I\right)\left(S_{\gamma}-i I\right)^{-1} g(\cdot)\right\rangle \\
& =\left\langle\left(S_{\gamma}+i I\right)\left(S_{\gamma}^{2}+I\right)^{-1} k(\cdot, w),\left(S_{\gamma}+i I\right) g(\cdot)\right\rangle \\
& =\left\langle\left(S_{\gamma}^{2}+I\right)^{-1} k(\cdot, w), g(\cdot)\right\rangle_{+2}
\end{aligned}
$$

for all $g(z) \in \mathcal{B}_{+2}^{(\gamma)}$ and $w \in \mathbb{C}$, it follows that

$$
k_{+2}(z, w)=\left(S_{\gamma}^{2}+I\right)^{-1} k(z, w) .
$$

Let us introduce a second scale of spaces associated with $\mathcal{B}$ and $S$. Define $\mathcal{F}_{+1}:=\operatorname{dom}\left(S^{*}\right)$ equipped with the graph inner product

$$
\langle f, g\rangle_{+\mathcal{F}}:=\left\langle S^{*} f, S^{*} g\right\rangle+\langle f, g\rangle .
$$

Due to (11a), for $f(z)$ and $g(z)$ in $\operatorname{dom}\left(S^{*}\right)$, one has

$$
\begin{array}{ll}
f(z)=h(z)+b S_{\gamma}\left(S_{\gamma}-i I\right)^{-1} k(z, i), & h(z) \in \operatorname{dom}\left(S_{\gamma}\right), \quad b \in \mathbb{C}, \\
g(z)=n(z)+d S_{\gamma}\left(S_{\gamma}-i I\right)^{-1} k(z, i), & n(z) \in \operatorname{dom}\left(S_{\gamma}\right), \quad d \in \mathbb{C} .
\end{array}
$$

From these expansions, using (11b), one obtains

$$
\langle f, g\rangle_{+\mathcal{F}}=\langle h, n\rangle_{+2}+\bar{b} d\left\|\left(S_{\gamma}-i\right)^{-1} k(\cdot, i)\right\|_{+2}^{2}=\langle h, n\rangle_{+2}+\bar{b} d\|k(\cdot, i)\|^{2} .
$$

This clearly implies $\|f\|_{+\mathcal{F}}=\|f\|_{+2}$ whenever $f \in \operatorname{dom}\left(S_{\gamma}\right)$, which gives rise to the scale

$$
\mathcal{B}_{+2}^{(\gamma)} \subset \mathcal{F}_{+1} \subset \mathcal{B} \subset \mathcal{F}_{-1} \subset \mathcal{B}_{-2}^{(\gamma)}
$$

here $\mathcal{F}_{-1}$ is the completion of $\mathcal{B}$ with respect to the norm

$$
\|f\|_{-\mathcal{F}}:=\sup _{g(z) \in \operatorname{dom}\left(S^{*}\right) \backslash\{0\}} \frac{|\langle f, g\rangle|}{\|g\|_{+\mathcal{F}}} .
$$

Remark $3 . \mathcal{B}_{+2}^{(\gamma)}$ is not dense in $\mathcal{F}_{+1}$, a fact obvious upon inspection of (15). 
For the next result we will use the following consequence of Lemma 2.1,

$$
\begin{aligned}
U\left(\overline{w_{0}}\right) k(v, z) & =\left(S^{*}-w_{0} I\right)\left(S_{\gamma}-\overline{w_{0}} I\right)^{-1} k(v, z) \\
& =\frac{\bar{z}-w_{0}}{\bar{z}-\overline{w_{0}}} k(v, z)-\frac{\overline{w_{0}}-w_{0}}{\bar{z}-\overline{w_{0}}} \frac{s_{\gamma}(\bar{z})}{s_{\gamma}\left(\overline{w_{0}}\right)} k\left(v, w_{0}\right), \quad z \neq w_{0},
\end{aligned}
$$

where $U(w)$ is the Cayley transform (9).

Theorem 3.2. $\mathcal{F}_{+1}$ is a Hilbert space of entire functions with reproducing kernel, isometrically invariant under the conjugation $f(z) \mapsto f^{\#}(z):=\overline{f(\bar{z})}$, and invariant under the mapping $f(z) \mapsto \frac{z-\bar{w}}{z-w} f(z)$ whenever $w \in \mathbb{C}$ is a non real zero of $f(z)$.

Proof. Given $f(z), g(z) \in \operatorname{dom}\left(S^{*}\right)$ and $w \in \mathbb{C}$,

$$
|g(w)-f(w)|=|\langle k(\cdot, w), g(\cdot)-f(\cdot)\rangle| \leq\|k(\cdot, w)\|_{-\mathcal{F}}\|g-f\|_{+\mathcal{F}},
$$

which implies that point evaluation is continuous in $\mathcal{F}_{+1}$.

Next, suppose $f(z) \in \operatorname{dom}\left(S^{*}\right)$. Then, according to (5a),

$$
f(z)=h(z)+a^{(+)} k(z, \bar{w})+a^{(-)} k(z, w),
$$

for some $h(z) \in \operatorname{dom}(S), a, b \in \mathbb{C}$ and some fixed $w \in \mathbb{C}_{+}$. Thus

$$
f^{\#}(z)=h^{\#}(z)+\overline{a^{(+)} k(\bar{z}, \bar{w})}+\overline{a^{(-)} k(\bar{z}, w)} .
$$

From this equality, one concludes that $f^{\#}(z) \in \operatorname{dom}\left(S^{*}\right)$ since $\overline{k(\bar{z}, w)}=k(z, \bar{w})$ and $h^{\#}(z) \in \operatorname{dom}(S)$. Moreover, recalling that $S^{*}$ commutes with the conjugation ${ }^{\#}$, one obtains

$$
\left\|g^{\#}\right\|_{+\mathcal{F}}^{2}=\left\|S^{*} g^{\#}\right\|^{2}+\left\|g^{\#}\right\|^{2}=\left\|\left(S^{*} g\right)^{\#}\right\|^{2}+\|g\|^{2}=\|g\|_{+\mathcal{F}}^{2} .
$$

Finally, suppose that $f(z) \in \operatorname{dom}\left(S^{*}\right)$ has a non real zero $w_{0}$. Define $g(z):=$ $U\left(w_{0}\right) f(z)$, where $U(w)$ is given in $(9)$. Since $U(w)$ maps $\operatorname{dom}\left(S^{*}\right)$ into itself, the function $g(z)$ belongs to $\operatorname{dom}\left(S^{*}\right)$. Moreover,

$$
\langle k(\cdot, z), g(\cdot)\rangle=\left\langle U\left(\overline{w_{0}}\right) k(\cdot, z), f(\cdot)\right\rangle=\frac{z-\overline{w_{0}}}{z-w_{0}} f(z),
$$

as implied by (16). 
In general, $\mathcal{F}_{+1}$ is not a $\mathrm{dB}$ space. This is somewhat hinted in the proof above: If $f(z) \in \operatorname{dom}\left(S^{*}\right)$ has a non real zero $w_{0}$, then $\left(z-\overline{w_{0}}\right)\left(z-w_{0}\right)^{-1} f(z) \in$ $\operatorname{dom}\left(S^{*}\right)$ but $\left\|\left(\cdot-\overline{w_{0}}\right)\left(\cdot-w_{0}\right)^{-1} f\right\|_{+\mathcal{F}}$ does not necessarily equal $\|f\|_{+\mathcal{F}}$. The next (counter) example illustrates this fact.

Example 3.3. Consider the Paley-Wiener space

$$
\mathcal{P} \mathcal{W}_{a}:=\left\{f(z)=\int_{-a}^{a} e^{i z x} \varphi(x) d x: \varphi \in L_{2}(-a, a)\right\},
$$

whose norm clearly obeys $\|f\|_{\mathcal{P} \mathcal{W}_{a}}=\|\varphi\|_{L_{2}(-a, a)}$. Moreover, the Fourier transform is a unitary mapping between the maximally defined operator $\varphi \mapsto i \varphi^{\prime}$ in $L_{2}(-a, a)$ and $S^{*}$, thus

$$
\operatorname{dom}\left(S^{*}\right)=\left\{f(z)=\int_{-a}^{a} e^{i z x} \varphi(x) d x: \varphi \in \mathrm{AC}[-a, a]\right\} .
$$

It follows that

$$
\|f\|_{+\mathcal{F}}^{2}=\left\|\varphi^{\prime}\right\|_{L_{2}(-a, a)}^{2}+\|\varphi\|_{L_{2}(-a, a)}^{2} .
$$

Its selfadjoint restrictions $S_{\gamma}, \gamma \in[0, \pi)$, have domains

$$
\operatorname{dom}\left(S_{\gamma}\right)=\left\{f(z)=\int_{-a}^{a} e^{i z x} \varphi(x) d x: \varphi \in \mathrm{AC}[-a, a] \text { and } \varphi(a)=e^{2 i \gamma} \varphi(-a)\right\} .
$$

Suppose $f(z)=\operatorname{dom}\left(S^{*}\right)$ has a non real zero $w_{0}$. If $f(z)$ is the Fourier transform of $\varphi \in L_{2}(-a, a)$, one has

$$
\frac{z-\overline{w_{0}}}{z-w_{0}} f(z)=\int_{-a}^{a} e^{i z x} \eta(x) d x
$$

where (choosing $\gamma=0$ )

$$
\eta(x)=\left(A_{0}-\overline{w_{0}} I\right)\left(A_{0}-w_{0} I\right)^{-1} \varphi(x)=\varphi(x)-2 i \operatorname{re}\left(w_{0}\right) \int_{-a}^{x} e^{i w_{0}(y-x)} \varphi(y) d y .
$$

Now set $\varphi(x)=e^{-x}$, whose image is

$$
f(z)= \begin{cases}\frac{2 \sin (z+i) a}{z+i}, & z \neq-i, \\ 2 a, & z=-i\end{cases}
$$


and choose $w_{0}=\pi / a-i$ so

$$
\eta(x)=e^{-x}+i \frac{2 a}{\pi} e^{-x}\left(1+e^{-i \pi x / a}\right)
$$

One can readily verify that $\left\|\varphi^{\prime}\right\|_{L_{2}(-a, a)} \neq\left\|\eta^{\prime}\right\|_{L_{2}(-a, a)}$.

Denote by $\langle\cdot, \cdot\rangle_{\mathcal{F}}$ the pairing between $\mathcal{F}_{-1}$ and $\mathcal{F}_{+1}$. The expression $\langle f, \phi\rangle_{\mathcal{F}}$ for $f \in \mathcal{F}_{+}$and $\phi \in \mathcal{F}_{-1}$ of course means $\overline{\langle\phi, f\rangle}_{\mathcal{F}}$.

Proposition 3.4. The mapping $\langle k(\cdot, z), \cdot\rangle_{\mathcal{F}}$ is a bijection between $\mathcal{F}_{-1}$ and assoc $\mathcal{B}$.

Proof. Consider $\psi \in \mathcal{F}_{-1}$ and define

$$
f(z):=\langle k(\cdot, z), \psi\rangle_{\mathcal{F}} .
$$

Given $w \in \mathbb{C}$, choose $\gamma$ such that $w \notin \operatorname{spec}\left(S_{\gamma}\right)$. For any $g(z) \in \mathcal{B}$,

$$
\begin{aligned}
\frac{f(z) g(w)-f(w) g(z)}{z-w} & =\frac{1}{z-w}\langle k(\cdot, z), g(w) \psi-f(w) g\rangle_{\mathcal{F}} \\
& =\left\langle\frac{k(\cdot, z)-k(\cdot, w)}{\bar{z}-\bar{w}}, g(w) \psi-f(w) g\right\rangle_{\mathcal{F}} \\
& =\left\langle R_{\gamma}(w) k(\cdot, z), g(w) \psi-f(w) g\right\rangle_{\mathcal{F}} \\
& =\langle k(\cdot, z), n(\cdot, w)\rangle,
\end{aligned}
$$

where $n(z, w):=g(w) \tilde{R}_{\gamma}(w) \psi(z)-f(w) R_{\gamma}(w) g(z)$; Lemma 2.1 has been used in the computation above. Since $n(z, w) \in \mathcal{B}$ (as a function of $z$ ), it follows that $f(z) \in \operatorname{assoc} \mathcal{B}$. Therefore, $\langle k(\cdot, z), \cdot\rangle_{\mathcal{F}} \operatorname{maps} \mathcal{F}_{-1}$ into assoc $\mathcal{B}$.

Suppose now $f(z) \in \operatorname{assoc} \mathcal{B}$, that is, $f(z)=z g(z)+h(z)$ for some $g(z), h(z) \in$ $\mathcal{B}$. Consider $\left\{g_{n}(z)\right\}_{n \in \mathbb{N}} \subset \operatorname{dom}(S)$ such that $\left\|g-g_{n}\right\| \rightarrow 0$ and define $f_{n}(z):=$ $z g_{n}(z)+h(z)$. Since

$$
\frac{\left|\left\langle f_{n}-f_{m}, l\right\rangle\right|}{\|l\|_{+\mathcal{F}}}=\frac{\left|\left\langle g_{n}-g_{m}, S^{*} l\right\rangle\right|}{\|l\|_{+\mathcal{F}}} \leq\left\|g_{n}-g_{m}\right\|
$$

for any $l(z) \in \operatorname{dom}\left(S^{*}\right)$, it follows that $\left\{f_{n}(z)\right\}_{n \in \mathbb{N}}$ is Cauchy convergent in $\mathcal{F}_{-1}$. Let $\phi \in \mathcal{F}_{-1}$ be its limit, which is well defined (i. e., independent of the sequence) by standard arguments. Since $k(z, w) \in \operatorname{dom}\left(S^{*}\right)$ for all $w \in \mathbb{C}$, we can define 
$\tilde{f}(z):=\langle k(\cdot, z), \phi\rangle_{\mathcal{F}}$. Now, on one hand we have

$$
\left|\tilde{f}(z)-f_{n}(z)\right|=\left|\left\langle k(\cdot, z), \phi-f_{n}\right\rangle_{\mathcal{F}}\right| \leq\|k(\cdot, z)\|_{+\mathcal{F}}\left\|\phi-f_{n}\right\|_{-\mathcal{F}} .
$$

On the other hand, we have assoc $\mathcal{B}=\mathcal{B}\left(e_{1}\right)$ where we can choose $e_{1}(z)=$ $(z+i) e(z)$. Let $k_{1}(z, w)$ be the reproducing kernel in $\mathcal{B}\left(e_{1}\right)$ [17]. Then,

$$
\begin{aligned}
\left|f(z)-f_{n}(z)\right|^{2} & \leq\left\|k_{1}(\cdot, z)\right\|_{\mathcal{B}\left(e_{1}\right)}^{2}\left\|f-f_{n}\right\|_{\mathcal{B}\left(e_{1}\right)}^{2} \\
& =k_{1}(z, z) \int_{\mathbb{R}} \frac{\left|x g(x)-x g_{n}(x)\right|^{2}}{\left(x^{2}+1\right)|e(x)|^{2}} d x \\
& \leq k_{1}(z, z)\left\|g-g_{n}\right\|^{2} .
\end{aligned}
$$

Therefore,

$$
|\tilde{f}(z)-f(z)| \leq\left|\tilde{f}(z)-f_{n}(z)\right|+\left|f(z)-f_{n}(z)\right|
$$

can be made arbitrarily small in compact subsets of $\mathbb{C}$ due to (18) and (19). This in fact shows that $\langle k(\cdot, z), \cdot\rangle_{\mathcal{F}}: \mathcal{F}_{-1} \rightarrow$ assoc $\mathcal{B}$ is onto. Since $\langle k(\cdot, z), \phi\rangle_{\mathcal{F}} \equiv 0$, one has $\phi=0$. Thus this mapping is also injective.

On $\operatorname{assoc} \mathcal{B}$ - and with some abuse of notation - define the norm

$$
\|f\|_{-\mathcal{F}}:=\|\psi\|_{-\mathcal{F}}
$$

with $f(z)$ and $\psi$ related as in (17). This makes $\langle k(\cdot, z), \cdot\rangle_{\mathcal{F}}: \mathcal{F}_{-1} \rightarrow \operatorname{assoc} \mathcal{B}$ an isometry and, taking into account the underlying inner product, assoc $\mathcal{B}$ is a Hilbert space.

Remark 4. In the terminology of [23], assoc $\mathcal{B}$ is just a dB-normable linear space of entire functions. It is well known that, if $\mathcal{B}=\mathcal{B}(e(z))$ for some $e(z) \in \mathcal{H B}$, then assoc $\mathcal{B}=\mathcal{B}((z+w) e(z))$ setwise, for any $w \in \mathbb{C}_{+}$. The next theorem shows that $(20)$ provides a different norm under which assoc $\mathcal{B}$ becomes a $\mathrm{dB}$ space.

Theorem 3.5. The Hilbert space assoc $\mathcal{B}$ given above, i. e. with the norm (20), is a dB space.

Proof. Consider $f(z), g(z) \in \operatorname{assoc} \mathcal{B}$. In accordance with (17), they are images of some $\psi, \eta \in \mathcal{F}_{-1}$. Given $w \in \mathbb{C}$,

$$
|f(w)-g(w)|=\left|\langle k(\cdot, w), \psi-\eta\rangle_{\mathcal{F}}\right| \leq\|k(\cdot, w)\|_{+\mathcal{F}}\|\psi-\eta\|_{-\mathcal{F}} .
$$

Then, the functional of point evaluation is continuous. 
Let ${ }^{\#}: \mathcal{F}_{-1} \rightarrow \mathcal{F}_{-1}$ be the extension of the conjugation $f(z) \mapsto f^{\#}(z)=\overline{f(\bar{z})}$ defined in $\mathcal{B}$, given by the rule

$$
\left\langle\psi^{\#}, f\right\rangle_{\mathcal{F}}:=\overline{\left\langle\psi, f^{\#}\right\rangle_{\mathcal{F}}}, \quad \psi \in \mathcal{F}_{-1}, \quad f(z) \in \operatorname{dom}\left(S^{*}\right) .
$$

Since assoc $\mathcal{B}$ coincides with $\mathcal{B}\left(e_{1}\right)$ setwise [17], if $f(z) \in \operatorname{assoc} \mathcal{B}$, then $f^{\#}(z) \in$ assoc $\mathcal{B}$. Indeed, if

$$
f(z)=\langle k(\cdot, z), \psi\rangle_{\mathcal{F}} \quad \text { then } \quad f^{\#}(z)=\left\langle k(\cdot, z), \psi^{\#}\right\rangle_{\mathcal{F}}
$$

this follows from the fact that $\overline{k(\bar{z}, w)}=k(z, \bar{w})$. Moreover,

$$
\begin{aligned}
\left\|f^{\#}\right\|_{-\mathcal{F}}=\left\|\psi^{\#}\right\|_{-\mathcal{F}} & =\sup _{g(z) \in \mathcal{F}_{+1} \backslash\{0\}} \frac{\left|\left\langle\psi^{\#}, g\right\rangle_{\mathcal{F}}\right|}{\|g\|_{+\mathcal{F}}} \\
& =\sup _{g(z) \in \mathcal{F}_{+1} \backslash\{0\}} \frac{\left|\left\langle\psi, g^{\#}\right\rangle_{\mathcal{F}}\right|}{\left\|g^{\#}\right\|_{+\mathcal{F}}}=\|\psi\|_{-\mathcal{F}}=\|f\|_{-\mathcal{F}} .
\end{aligned}
$$

Finally, let us consider the mapping (1). Suppose $f(z)=\langle k(\cdot, z), \psi\rangle_{\mathcal{F}} \in \operatorname{assoc} \mathcal{B}$ such that $f\left(w_{0}\right)=0$ for some $w_{0} \in \mathbb{C} \backslash \mathbb{R}$. Let $\hat{U}(w): \mathcal{F}_{-1} \rightarrow \mathcal{F}_{-1}$ denote the dual of the Cayley transform $U(\bar{w}): \mathcal{F}_{+1} \rightarrow \mathcal{F}_{+1}$. Due to (16),

$$
\left\langle k(\cdot, z), \hat{U}\left(w_{0}\right) \psi\right\rangle_{\mathcal{F}}=\left\langle U\left(\overline{w_{0}}\right) k(\cdot, z), \psi\right\rangle_{\mathcal{F}}=\frac{z-\overline{w_{0}}}{z-w_{0}} f(z)
$$

Furthermore,

$$
\begin{aligned}
\left\|\hat{U}\left(\overline{w_{0}}\right) \psi\right\|_{-\mathcal{F}}=\sup _{g(z) \in \mathcal{F}_{+1} \backslash\{0\}} \frac{\left|\left\langle\hat{U}\left(\overline{w_{0}}\right) \psi, g\right\rangle_{\mathcal{F}}\right|}{\|g\|_{+\mathcal{F}}} & =\sup _{g(z) \in \mathcal{F}_{+1} \backslash\{0\}} \frac{\left|\left\langle\psi, U\left(w_{0}\right) g\right\rangle_{\mathcal{F}}\right|}{\left\|U\left(\overline{w_{0}}\right) U\left(w_{0}\right) g\right\|_{+\mathcal{F}}} \\
& =\sup _{g(z) \in \mathcal{F}_{+1} \backslash\{0\}} \frac{\left|\langle\psi, g\rangle_{\mathcal{F}}\right|}{\left\|U\left(\overline{w_{0}}\right) g\right\|_{+\mathcal{F}}},
\end{aligned}
$$

where the last equation arises from $U(w): \mathcal{F}_{+1} \rightarrow \mathcal{F}_{+1}$ being onto (see (9)). Since $U(w)$ is also an isometry in there,

$$
\left\|\frac{(\cdot)-\overline{w_{0}}}{(\cdot)-w_{0}} f(\cdot)\right\|_{-\mathcal{F}}=\left\|\hat{U}\left(\overline{w_{0}}\right) \psi\right\|_{-\mathcal{F}}=\|\psi\|_{-\mathcal{F}}=\|f\|_{-\mathcal{F}},
$$

thereby completing the proof. 
In view of Proposition 3.4 and Theorem 3.5, we henceforth identify $\mathcal{F}_{-1}$ with its realization as the Hilbert space assoc $\mathcal{B}$ which is equipped with the norm (20). Following up this identification, we define

$$
\langle f, g\rangle_{\mathcal{F}}:=\langle\psi, g\rangle_{\mathcal{F}}, \quad f(z) \in \operatorname{assoc} \mathcal{B}, \quad g(z) \in \operatorname{dom}\left(S^{*}\right),
$$

where $\psi \in \mathcal{F}_{-1}$ is related to $f(z)$ by (17). In particular,

$$
\langle k(\cdot, z), f\rangle_{\mathcal{F}}=f(z), \quad f(z) \in \mathcal{F}_{-1} .
$$

The next result provides an explicit formula for the duality pairing between $\mathcal{F}_{+1}$ and $\mathcal{F}_{-1}$.

Proposition 3.6. Suppose $\psi \in \mathcal{F}_{-1}$ and $f(z) \in \operatorname{assoc} \mathcal{B}$ be such that $f(z)=$ $\langle k(\cdot, z), \psi\rangle_{\mathcal{F}}$. Then, for every $g(z) \in \mathcal{F}_{+1}$,

$$
\langle\psi, g\rangle_{\mathcal{F}}=\int_{\mathbb{R}} \frac{\overline{f(x)} h(x)}{|e(x)|^{2}} d x+a^{(+)} f^{\#}(i)+a^{(-)} f^{\#}(-i),
$$

where $g(z)=h(z)+a^{(+)} k(z,-i)+a^{(-)} k(z, i)$ is decomposed in accordance with (5a).

Proof. We have $\mathcal{F}_{-1}=\mathcal{B}\left(e_{\mathcal{F}}\right)$ for some $e_{\mathcal{F}}(z) \in \mathcal{H B}$. Also, since $\mathcal{B}=\mathcal{B}(e)$ for some $e(z) \in \mathcal{H B}$ free of real zeros (see Remark 1$), \mathcal{F}_{-1}=\mathcal{B}\left(e_{1}\right)$ setwise with $e_{1}(z):=(z+i) e(z)$. Thus, if $\mathfrak{I}(g)$ denotes the right hand side of $(21)$, then

$$
\begin{aligned}
|\mathfrak{I}(g)| & \leq\|f\|_{\mathcal{B}\left(e_{1}\right)}\|h\|_{+2}+\left|a^{(+)}\right|\|f\|_{-\mathcal{F}}\|k(\cdot,-i)\|_{+\mathcal{F}}+\left|a^{(-)}\right|\|f\|_{-\mathcal{F}}\|k(\cdot, i)\|_{+\mathcal{F}} \\
& \leq C\|f\|_{-\mathcal{F}}\left(\|h\|_{+2}+\left(\left|a^{(+)}\right|+\left|a^{(-)}\right|\right)\|k(\cdot, i)\|_{+\mathcal{F}}\right),
\end{aligned}
$$

where the second inequality is implied by the fact that the norms induced by $e_{\mathcal{F}}$ and $e_{1}$ are equivalent. Now, since

$$
\|g\|_{+\mathcal{F}}^{2}=\|h\|_{+2}^{2}+\left(\left|a^{(+)}\right|^{2}+\left|a^{(-)}\right|^{2}\right)\|k(\cdot, i)\|_{+\mathcal{F}}^{2},
$$

it follows that, for some possibly different $C>0$,

$$
\left|\langle f, g\rangle_{\mathcal{F}}\right| \leq C\|f\|_{-\mathcal{F}}\|g\|_{+\mathcal{F}}
$$

so $\mathfrak{I}$ defines a continuous functional on $\mathcal{F}_{+1}$. Finally, it is easy to verify that $\mathfrak{I}$ coincides with $\langle f, \cdot\rangle$ whenever $f(z) \in \mathcal{B}$ so it is equal to $\langle\psi, \cdot\rangle_{\mathcal{F}}$. 
Let $\mathcal{M}_{0}^{(\gamma)} \subset \mathcal{F}_{-1}$ be the annihilator of $\operatorname{dom}\left(S_{\gamma}\right)$, that is,

$$
\mathcal{M}_{0}^{(\gamma)}:=\left\{g(z) \in \operatorname{assoc} \mathcal{B}:\langle g, h\rangle_{\mathcal{F}}=0 \text { for all } h(z) \in \operatorname{dom}\left(S_{\gamma}\right)\right\}
$$

Theorem 3.7. Every element $\mathfrak{F} \in \operatorname{assoc} \mathcal{B} / \mathcal{M}_{0}^{(\gamma)}$ defines a unique element in $\mathcal{B}_{-2}^{(\gamma)}$ by the rule

$$
\langle\mathfrak{F}, h\rangle_{2}:=\langle f, h\rangle_{\mathcal{F}}, \quad h(z) \in \mathcal{F}_{+1}
$$

where $f(z) \in \operatorname{assoc} \mathcal{B}$ is any representative of $\mathfrak{F}$. Conversely, to every $\varphi \in \mathcal{B}_{-2}^{(\gamma)}$ there corresponds a unique $\mathfrak{F} \in \operatorname{assoc} \mathcal{B} / \mathcal{M}_{0}^{(\gamma)}$ such that

$$
\langle\varphi, h\rangle_{2}=\langle\mathfrak{F}, h\rangle_{2}
$$

in the sense of (22).

Proof. The trueness of the first part of the statement is rather obvious, since for every element $g(z)$ of $\operatorname{dom}\left(S_{\gamma}\right)$ one has $\|g\|_{+2}=\|g\|_{+\mathcal{F}}$. Indeed,

$$
\|\mathfrak{F}\|_{-2}=\sup _{g(z) \in \operatorname{dom}\left(S_{\gamma}\right) \backslash\{0\}} \frac{\left|\langle f, g\rangle_{\mathcal{F}}\right|}{\|g\|_{+\mathcal{F}}} \leq\|f\|_{-\mathcal{F}},
$$

for all $f(z)$ within the equivalence class $\mathfrak{F}$.

As for the second part of the statement, consider $\varphi \in \mathcal{B}_{-2}^{(\gamma)}$. Given $c \in \mathbb{C}$, define $\varphi_{c} \in \mathcal{F}_{-1}$ by the rule

$$
\left\langle\varphi_{c}, g\right\rangle_{\mathcal{F}}:=\langle\varphi, h\rangle_{2}+\bar{c} b
$$

where $h(z) \in \operatorname{dom}\left(S_{\gamma}\right)$ and $b \in \mathbb{C}$ are related to $g(z) \in \operatorname{dom}\left(S^{*}\right)$ by the decomposition defining (11a) [3, Lemma 1.3.1]. By Proposition 3.4, $\varphi_{c}$ can be identified with some $f_{c}(z) \in \operatorname{assoc} \mathcal{B}$. Clearly,

$$
\left\langle f_{c}, \cdot\right\rangle_{\mathcal{F}} \Upsilon_{\operatorname{dom}\left(S_{\gamma}\right)}=\langle\varphi, \cdot\rangle_{2} .
$$

Thus, the associated element in assoc $\mathcal{B} / \mathcal{M}_{0}^{(\gamma)}$ is $\mathfrak{F}=\left\{f_{c}(z): c \in \mathbb{C}\right\}$.

\section{Singular rank-one perturbations}

Let us turn to singular rank-one perturbations of selfadjoint extensions of the operator $S$. To keep the notation simple, let us fix $\gamma=\pi / 2$. We show below that the "correct" perturbation of the operator $S_{\pi / 2}$ is performed along the function 
$s_{0}(z)$ in the sense that $S$ is precisely $S_{\pi / 2}$ restricted to those $f(z) \in \operatorname{dom}\left(S_{\pi / 2}\right)$ that obey $\left\langle s_{0}, f\right\rangle_{2}=0$.

Lemma 4.1. Assume $\mu \in \operatorname{spec}\left(S_{\pi / 2}\right)$. Then,

$$
\langle k(\cdot, \mu), g\rangle_{2}=g(\mu)
$$

for every $g(z) \in \operatorname{assoc} \mathcal{B}$.

Proof. Given $g(z) \in \operatorname{assoc} \mathcal{B}$, let $\left\{h_{l}(z)\right\} \subset \mathcal{B}$ be any sequence converging to it in $\mathcal{B}_{-2}^{(\pi / 2)}$. Then, recalling that $k(z, \mu) \in \operatorname{dom}\left(S_{\pi / 2}\right)$, one has

$$
\left|h_{l}(\mu)-h_{m}(\mu)\right|=\left|\left\langle k(\cdot, \mu),\left(h_{l}-h_{m}\right)\right\rangle_{2}\right| \leq\|k(\cdot, \mu)\|_{+2}\left\|h_{l}-h_{m}\right\|_{-2}
$$

so $\left\{h_{l}(\mu)\right\}$ is convergent. By standard arguments the limit equals $g(\mu)$.

Lemma 4.2. For every $w \notin \operatorname{spec}\left(S_{\pi / 2}\right)$,

$$
\hat{R}_{\pi / 2}(w) s_{0}(z)=-\frac{\pi}{s_{\pi / 2}(w)} k(z, \bar{w})
$$

Proof. We need to show that

$$
\left\langle\hat{R}_{\pi / 2}(w) s_{0}(\cdot), f(\cdot)\right\rangle=-\frac{\pi}{s_{\pi / 2}(\bar{w})} f(\bar{w})
$$

for every $f(z) \in \mathcal{B}$. Due to the continuity of the inner product, it will suffice to show the assertion on elements of a basis of $\mathcal{B}$. Thus, let us consider $k\left(z, \mu_{n}\right)$ with $\mu_{n} \in \operatorname{spec}\left(S_{\pi / 2}\right)$. We have

$$
\begin{aligned}
\left\langle\hat{R}_{\pi / 2}(w) s_{0}(\cdot), k\left(\cdot, \mu_{n}\right)\right\rangle & =\left\langle s_{0}(\cdot),\left(S_{\pi / 2}-\bar{w} I\right)^{-1} k\left(\cdot, \mu_{n}\right)\right\rangle_{2} \\
& =\left\langle s_{0}(\cdot),\left(\mu_{n}-\bar{w}\right)^{-1} k\left(\cdot, \mu_{n}\right)\right\rangle_{2} \\
& =\left(\mu_{n}-\bar{w}\right)^{-1}\left\langle s_{0}(\cdot), k\left(\cdot, \mu_{n}\right)\right\rangle_{2} \\
& =\left(\mu_{n}-\bar{w}\right)^{-1} s_{0}\left(\mu_{n}\right),
\end{aligned}
$$

where we have used the previous lemma and the fact that $s_{0}(z)$ is real entire.

Recalling (4) we have

$$
k\left(\bar{w}, \mu_{n}\right)=\frac{s_{\pi / 2}(\bar{w}) s_{0}\left(\mu_{n}\right)}{\pi\left(\bar{w}-\mu_{n}\right)}
$$


whence the assertion follows.

Lemma 4.3. The function $s_{0}(z)$ is a generating element (cyclic in the terminology of [8]) of $S_{\pi / 2}$.

Proof. Since $S$ is simple and $k(z, \bar{w}) \in \operatorname{ker}\left(S^{*}-w I\right), \mathcal{B}$ is the closure of

$$
\underset{w \in \mathbb{C} \backslash \mathbb{R}}{\operatorname{span}}\left\{\operatorname{ker}\left(S^{*}-w I\right)\right\}=\operatorname{span}_{w \in \mathbb{C} \backslash \mathbb{R}}\{k(z, \bar{w})\}
$$

This identity along with Lemma 4.2 implies that

$$
\operatorname{span}_{w \in \mathbb{C} \backslash \mathbb{R}}\left\{\hat{R}_{\pi / 2}(w) s_{0}(z)\right\}
$$

is a total set in $\mathcal{B}$.

Proposition 4.4. Define $\mathcal{D}_{0}:=\left\{f(z) \in \operatorname{dom}\left(S_{\pi / 2}\right):\left\langle s_{0}, f\right\rangle_{2}=0\right\}$. Then,

$$
S=S_{\pi / 2} \Upsilon_{\mathcal{D}_{0}}
$$

Proof. First, choose any $f(z) \in \operatorname{dom}(S)$ and set $g(z):=(z+i) f(z)$; clearly $g(z) \in \mathcal{B}$. Then, according to (13),

$$
\begin{aligned}
\left\langle s_{0}, f\right\rangle_{2} & =\left\langle\hat{R}_{\pi / 2}(i) s_{0}(\cdot),\left(S_{\pi / 2}+i I\right) f(\cdot)\right\rangle \\
& =-\frac{\pi}{s_{\pi / 2}(-i)}\langle k(\cdot,-i), g(\cdot)\rangle \\
& =g(-i)=0
\end{aligned}
$$

Therefore, $\operatorname{dom}(S) \subset \mathcal{D}_{0}$.

Now suppose $f(z) \in \mathcal{D}_{0}$. Since $f(z) \in \operatorname{dom}\left(S_{\pi / 2}\right)$, it follows from (6) that

$$
\left(S_{\pi / 2}+i I\right) f(z)=(z+i) f(z)+\frac{s_{\pi / 2}(z)}{s_{\pi / 2}(-i)} g(-i)
$$

where $g(z) \in \mathcal{B}$ satisfies

$$
f(z)=\frac{g(z)-\frac{s_{\pi / 2}(z)}{s_{\pi / 2}(-i)} g(-i)}{z+i}
$$


A computation like the one above yields

$$
\left\langle s_{0}, f\right\rangle_{2}=\left\langle\hat{R}_{\pi / 2}(i) s_{0}(\cdot),\left(S_{\pi / 2}+i I\right) f(\cdot)\right\rangle=g(-i)
$$

so the assumption $\left\langle s_{0}, f\right\rangle_{2}=0$ implies $g(-i)=0$ in turn implying $f(z) \in$ $\operatorname{dom}(S)$. This completes the proof.

Proposition 4.4 implies that any other selfadjoint $S_{\gamma}$ of $S$ is related to $S_{\pi / 2}$ by Krein's formula [3, Theorem 1.2.1],

$$
R_{\gamma}(w)-R_{\pi / 2}(w)=\frac{1}{\lambda-q(w)}\left\langle\hat{R}_{\pi / 2}(\bar{w}) s_{0}(\cdot), \cdot\right\rangle \hat{R}_{\pi / 2}(w) s_{0}(z)
$$

with $w: \operatorname{im}(w) \neq 0$, where

$$
q(w):=\left\langle\hat{R}_{\pi / 2}(i) s_{0}(\cdot),\left(I+w S_{\pi / 2}\right) R_{\pi / 2}(w) \hat{R}_{\pi / 2}(i) s_{0}(\cdot)\right\rangle
$$

is the Krein's $Q$-function. Below we find the relation between $\lambda$ and $\gamma$.

Lemma 4.5. Let $q(w)$ be the function defined by (24). Then,

$$
q(w)=\pi \operatorname{re}\left(\frac{s_{0}(i)}{s_{\pi / 2}(i)}\right)-\pi \frac{s_{0}(w)}{s_{\pi / 2}(w)}
$$

Proof. Since $\left(I+w S_{\pi / 2}\right)\left(S_{\pi / 2}-w I\right)^{-1}=w I+\left(w^{2}+1\right)\left(S_{\pi / 2}-w I\right)^{-1}$, it follows from Lemma 4.2 that

$$
q(w)=\frac{\pi^{2}}{\left|s_{\pi / 2}(i)\right|^{2}} w k(i, i)+\frac{\pi^{2}}{\left|s_{\pi / 2}(i)\right|^{2}}\left(w^{2}+1\right)\left\langle k(\cdot,-i),\left(S_{\pi / 2}-w I\right)^{-1} k(\cdot,-i)\right\rangle .
$$

Resorting to Lemma 2.1, one rewrites this as follows

$$
q(w)=\frac{\pi^{2}}{\left|s_{\pi / 2}(i)\right|^{2}}(w+i) \frac{s_{\pi / 2}(i)}{s_{\pi / 2}(w)} k(w, i)-\frac{\pi^{2}}{\left|s_{\pi / 2}(i)\right|^{2}} i k(i, i) .
$$

Finally, a computation involving (4) yields (25).

Proposition 4.6. For $w \in \mathbb{C} \backslash \mathbb{R}$ one has

$$
R_{\gamma}(w)-R_{\pi / 2}(w)=\frac{1}{\pi \tan \gamma+\pi \frac{s_{0}(w)}{s_{\pi / 2}(w)}}\left\langle\hat{R}_{\pi / 2}(\bar{w}) s_{0}(\cdot), \cdot\right\rangle \hat{R}_{\pi / 2}(w) s_{0}(z) .
$$


Consequently, Krein's formula (23) holds true for $\lambda=\pi \tan \gamma+\pi \operatorname{re}\left(\frac{s_{0}(i)}{s_{\pi / 2}(i)}\right)$.

Proof. Let $f(z) \in \mathcal{B}$. By using (3), (6a), and Lemma 4.2 one obtains

$$
\begin{aligned}
\left(R_{\gamma}(w)-R_{\pi / 2}(w)\right) f(z) & =\frac{s_{\pi / 2}(z) s_{\gamma}(w)-s_{\pi / 2}(w) s_{\gamma}(z)}{z-w} \frac{f(w)}{s_{\pi / 2}(w) s_{\gamma}(w)} \\
& =\pi(\cos \gamma) k(z, \bar{w}) \frac{f(w)}{s_{\pi / 2}(w) s_{\gamma}(w)} \\
& =-\frac{\cos \gamma}{s_{\gamma}(w)} f(w) \hat{R}_{\pi / 2}(w) s_{0}(z) \\
& =\frac{\cos \gamma}{\pi} \frac{s_{\pi / 2}(w)}{s_{\gamma}(w)}\left\langle\hat{R}_{\pi / 2}(\bar{w}) s_{0}(\cdot), f(\cdot)\right\rangle \hat{R}_{\pi / 2}(w) s_{0}(z),
\end{aligned}
$$

whence the first statement follows. The second assertion is a consequence of Lemma 4.5.

Theorem 4.7. For $\beta \in[0, \pi) \backslash\{\pi / 2\}$, the following characterization holds

$$
\operatorname{dom}\left(S_{\gamma}\right)=\left\{\begin{array}{c}
g(z)=h(z)+b S_{\pi / 2} R_{\pi / 2}(-i) \hat{R}_{\pi / 2}(i) s_{0}(z) \\
h(z) \in \operatorname{dom}\left(S_{\pi / 2}\right), b \in \mathbb{C}:\left\langle s_{0}, h\right\rangle_{2}=\pi b\left(\tan \gamma+\operatorname{re} \frac{s_{0}(i)}{s_{\pi / 2}(i)}\right)
\end{array}\right\} .
$$

Proof. Let $\mathcal{D}$ denote the right hand side of the claimed identity. First, suppose $g(z) \in \operatorname{dom}\left(S_{\gamma}\right)$. According to $(6 \mathrm{a})$, for some unique $f(z) \in \mathcal{B}$, one has

$$
g(z)=\frac{f(z)-\frac{s_{\gamma}(z)}{s_{\gamma}(i)} f(i)}{z-i}=\frac{f(z)-\frac{s_{\pi / 2}(z)}{s_{\pi / 2}(i)} f(i)}{z-i}+\frac{\frac{s_{\pi / 2}(z)}{s_{\pi / 2}(i)}-\frac{s_{\gamma}(z)}{s_{\gamma}(i)}}{z-i} f(i) .
$$

The first term above belongs to $\operatorname{dom}\left(S_{\pi / 2}\right)$. As for the second term, we have

$$
\begin{aligned}
\text { 2nd term } & =\frac{\cos \gamma}{s_{\pi / 2}(i) s_{\gamma}(i)} \frac{s_{\pi / 2}(z) s_{0}(i)-s_{0}(z) s_{\pi / 2}(i)}{z-i} f(i) \\
& =\frac{\cos \gamma}{s_{\pi / 2}(i) s_{\gamma}(i)} \pi k(z,-i) f(i) \\
& =-\frac{\cos \gamma}{s_{\gamma}(i)} f(i) \hat{R}_{\pi / 2}(i) s_{0}(z) ;
\end{aligned}
$$


in this derivation we use (4) and Lemma 4.2. Now define

$$
h(z):=\frac{f(z)-\frac{s_{\pi / 2}(z)}{s_{\pi / 2}(i)} f(i)}{z-i}-i \frac{\cos \gamma}{s_{\gamma}(i)} f(i) R_{\pi / 2}(-i) \hat{R}_{\pi / 2}(i) s_{0}(z)
$$

clearly $h(z) \in \operatorname{dom}\left(S_{\pi / 2}\right)$. Then,

$$
\begin{aligned}
g(z) & =h(z)-\frac{\cos \gamma}{s_{\gamma}(i)} f(i)\left[\hat{R}_{\pi / 2}(i) s_{0}(z)-i R_{\pi / 2}(-i) \hat{R}_{\pi / 2}(i) s_{0}(z)\right] \\
& =h(z)+b S_{\pi / 2} R_{\pi / 2}(-i) \hat{R}_{\pi / 2}(i) s_{0}(z), \quad b:=-\frac{\cos \gamma}{s_{\gamma}(i)} f(i)
\end{aligned}
$$

Moreover,

$$
\begin{aligned}
\left\langle s_{0}, h\right\rangle_{2} & =\left\langle\hat{R}_{\pi / 2}(-i) s_{0}(\cdot), f(\cdot)\right\rangle+i b\left\langle\hat{R}_{\pi / 2}(i) s_{0}(\cdot), \hat{R}_{\pi / 2}(i) s_{0}(\cdot)\right\rangle \\
& =-\frac{\pi}{s_{\pi / 2}(i)} f(i)+i b \frac{\pi}{\left|s_{\pi / 2}(i)\right|^{2}} k(i, i) \\
& =\pi b\left(\frac{1}{\cos \gamma} \frac{s_{\gamma}(i)}{s_{\pi / 2}(i)}+i \frac{\frac{s_{0}(-i)}{s_{\pi / 2}(-i)}-\frac{s_{0}(i)}{s_{\pi / 2}(i)}}{2 i}\right) \\
& =\pi b\left(\tan \gamma+\operatorname{re} \frac{s_{0}(i)}{s_{\pi / 2}(i)}\right) .
\end{aligned}
$$

So far we have shown that $\operatorname{dom}\left(S_{\gamma}\right) \subset \mathcal{D}$. To prove that $\mathcal{D}$ is contained in $\operatorname{dom}\left(S_{\gamma}\right)$, it suffices to show that, for every $g(z) \in \mathcal{D}$, one has $g(z)=R_{\gamma}(i) l(z)$ for some $l(z) \in \mathcal{B}$. So let $g(z) \in \mathcal{D}$, i. e.,

$$
g(z)=h(z)+b S_{\pi / 2} R_{\pi / 2}(-i) \hat{R}_{\pi / 2}(i) s_{0}(z)
$$

with $h(z)=R_{\pi / 2}(i) f(z)$ and $b \in \mathbb{C}$ such that

$$
\left\langle s_{0}, h\right\rangle_{2}=\left\langle\hat{R}_{\pi / 2}(-i) s_{0}, f\right\rangle=-\frac{\pi}{s_{\pi / 2}(i)} f(i)=\pi b\left(\tan \gamma+\operatorname{re} \frac{s_{0}(i)}{s_{\pi / 2}(i)}\right) .
$$

Since $S_{\pi / 2} R_{\pi / 2}(-i)=I-i R_{\pi / 2}(-i)$ and $R_{\pi / 2}(-i) \hat{R}_{\pi / 2}(i)=R_{\pi / 2}(i) \hat{R}_{\pi / 2}(-i)$, one has

$$
g(z)=R_{\pi / 2}(i)\left(f(z)-i b \hat{R}_{\pi / 2}(-i) s_{0}(z)\right)+b \hat{R}_{\pi / 2}(i) s_{0}(z)
$$


Due to Lemma 4.2 and Proposition 4.6, the first term above becomes

$$
\begin{aligned}
& \text { 1st term }=R_{\gamma}(i)\left(f(z)+i \pi b \frac{k(z, i)}{s_{\pi / 2}(-i)}\right) \\
& \quad-\frac{1}{\tan \gamma+\frac{s_{0}(i)}{s_{\pi / 2}(i)}}\left\langle\hat{R}_{\pi / 2}(-i) s_{0}(\cdot), f(\cdot)-i b \hat{R}_{\pi / 2}(-i) s_{0}(\cdot)\right\rangle \hat{R}_{\pi / 2}(i) s_{0}(z) .
\end{aligned}
$$

However,

$$
\begin{aligned}
\left\langle\hat{R}_{\pi / 2}(-i) s_{0}(\cdot), f(\cdot)-i b \hat{R}_{\pi / 2}(-i) s_{0}(\cdot)\right\rangle & =-\frac{\pi}{s_{\pi / 2}} f(i)-i b \frac{\pi^{2}}{s_{\pi / 2}(i) s_{\pi / 2}(-i)} k(i, i) \\
& =\left\langle s_{0}, h\right\rangle_{2}+i \pi b \operatorname{im} \frac{s_{0}(i)}{s_{\pi / 2}(i)} \\
& =\pi b \tan \gamma+\pi b \frac{s_{0}(i)}{s_{\pi / 2}(i)}
\end{aligned}
$$

Thus,

$$
\text { 1st term }=R_{\gamma}(i)\left(f(z)+i \pi b \frac{k(z, i)}{s_{\pi / 2}(-i)}\right)-b \hat{R}_{\pi / 2}(i) s_{0}(z),
$$

which shows that $g(z) \in \operatorname{dom}\left(S_{\gamma}\right)$.

Lemma 4.8. For every $g(z) \in \operatorname{dom}\left(S^{*}\right)$,

$$
\left\langle s_{0}, g\right\rangle_{\mathcal{F}}=\left\langle s_{0}, h\right\rangle_{2}-\pi b \operatorname{re} \frac{s_{0}(i)}{s_{\pi / 2}(i)},
$$

with $h(z) \in \operatorname{dom}\left(S_{\pi / 2}\right)$ and $b \in \mathbb{C}$ related to $g(z)$ by the (unique) decomposition $g(z)=h(z)+b S_{\pi / 2} R_{\pi / 2}(-i) \hat{R}_{\pi / 2}(i) s_{0}(z)$.

Proof. An obvious computation shows that

$$
S_{\pi / 2} R_{\pi / 2}(-i) \hat{R}_{\pi / 2}(i)=\frac{1}{2} \hat{R}_{\pi / 2}(-i)+\frac{1}{2} \hat{R}_{\pi / 2}(i)
$$


Then, recalling Lemma 4.2,

$$
\begin{aligned}
\left\langle s_{0}, S_{\pi / 2} R_{\pi / 2}(-i) \hat{R}_{\pi / 2}(i)\right. & \left.s_{0}\right\rangle_{\mathcal{F}} \\
& =-\frac{\pi}{2 s_{\pi / 2}(-i)}\left\langle s_{0}, k(\cdot, i)\right\rangle_{\mathcal{F}}-\frac{\pi}{2 s_{\pi / 2}(i)}\left\langle s_{0}, k(\cdot,-i)\right\rangle_{\mathcal{F}} \\
& =-\pi \operatorname{re} \frac{s_{0}(i)}{s_{\pi / 2}(i)} .
\end{aligned}
$$

The assertion now follows straightforwardly.

For the statement of our last result below, we recall that $S_{\pi / 2}: \mathcal{B}_{+2}^{(\pi / 2)} \rightarrow \mathcal{B}$ has a unique contractive continuation $\hat{S}_{\pi / 2}: \mathcal{B} \rightarrow \mathcal{B}_{-2}^{(\pi / 2)}$.

Theorem 4.9. Let $\tilde{S}_{\pi / 2}$ be the restriction of $\hat{S}_{\pi / 2}$ to $\operatorname{dom}\left(S^{*}\right)$. For $\gamma \in(0, \pi)$, define $\tilde{S}_{\gamma}: \operatorname{dom}\left(S^{*}\right) \rightarrow \mathcal{B}_{-2}^{(\pi / 2)}$ by

$$
\tilde{S}_{\gamma}:=\tilde{S}_{\pi / 2}-\frac{\cot \gamma}{\pi}\left\langle s_{0}, \cdot\right\rangle_{\mathcal{F}} s_{0}(z)
$$

Then, $S_{\gamma}$ as described by (6) is the restriction of $\tilde{S}_{\gamma}$ to $\operatorname{dom}\left(S_{\gamma}\right)$.

Proof. If $g(z) \in \operatorname{dom}\left(S_{\gamma}\right)$, then

$$
g(z)=h(z)+b S_{\pi / 2} R_{\pi / 2}(-i) \hat{R}_{\pi / 2}(i) s_{0}(z)
$$

with $h(z) \in \operatorname{dom}\left(S_{\pi / 2}\right)$ and $b \in \mathbb{C}$, both related to each other in accordance with Theorem 4.7. Taking into account (29), one obtains

$$
\left\langle s_{0}, g\right\rangle_{\mathcal{F}}=\left\langle s_{0}, h\right\rangle_{2}-\pi b \text { re } \frac{s_{0}(i)}{s_{\pi / 2}(i)}=\pi b \tan \gamma
$$

Thus, so far,

$$
\tilde{S}_{\gamma} g(z)=S_{\pi / 2} h(z)+b \hat{S}_{\pi / 2} S_{\pi / 2} R_{\pi / 2}(-i) \hat{R}_{\pi / 2}(i) s_{0}(z)-b s_{0}(z) .
$$

Since

$$
\hat{S}_{\pi / 2} S_{\pi / 2} R_{\pi / 2}(-i) \hat{R}_{\pi / 2}(i)=\hat{I}-R_{\pi / 2}(-i) \hat{R}_{\pi / 2}(i)
$$

(here we use that $\left(\hat{S}_{\pi / 2}-i I\right) \hat{R}_{\pi / 2}(i)=\hat{I}$, the latter being the identity operator 
in $\mathcal{B}_{-2}^{(\pi / 2)}$, equation (30) becomes

$$
\begin{aligned}
\tilde{S}_{\gamma} g(z) & =S_{\pi / 2} h(z)-b R_{\pi / 2}(-i) \hat{R}_{\pi / 2}(i) s_{0}(z) \\
& =S_{\pi / 2} h(z)+i \frac{\pi b}{2}\left(\frac{k(z, i)}{s_{\pi / 2}(-i)}-\frac{k(z,-i)}{s_{\pi / 2}(i)}\right)
\end{aligned}
$$

the last equality follows from the resolvent identity (12) and Lemma 4.2. On the other hand, since $h(z)=R_{\pi / 2}(i) f(z)$ for some $f(z) \in \mathcal{B}$, one has (see (27) and (28))

$$
g(z)=R_{\gamma}(i)\left(f(z)+i \pi b \frac{k(z, i)}{s_{\pi / 2}(-i)}\right) .
$$

Therefore,

$$
\begin{aligned}
S_{\gamma} g(z)=z h(z)+ & z b S_{\pi / 2} R_{\pi / 2}(-i) \hat{R}_{\pi / 2}(i) s_{0}(z) \\
& +\frac{s_{\gamma}(z)}{s_{\gamma}(i)}\left(f(i)+i \pi b \frac{k(i, i)}{s_{\pi / 2}(-i)}\right) \\
=S_{\pi / 2} h(z) & -z \frac{\pi b}{2}\left(\frac{k(z, i)}{s_{\pi / 2}(-i)}+\frac{k(z,-i)}{s_{\pi / 2}(i)}\right) \\
& +\left(\frac{s_{\gamma}(z)}{s_{\gamma}(i)}-\frac{s_{\pi / 2}(z)}{s_{\pi / 2}(i)}\right) f(i)+i \pi b \frac{k(i, i)}{s_{\pi / 2}(-i)} \frac{s_{\gamma}(z)}{s_{\gamma}(i)} .
\end{aligned}
$$

Thus, it suffices to show that

$$
\begin{aligned}
0=-i \frac{\pi b}{2}\left(\frac{k(z, i)}{s_{\pi / 2}(-i)}-\right. & \left.\frac{k(z,-i)}{s_{\pi / 2}(i)}\right)-z \frac{\pi b}{2}\left(\frac{k(z, i)}{s_{\pi / 2}(-i)}+\frac{k(z,-i)}{s_{\pi / 2}(i)}\right) \\
& +\left(\frac{s_{\gamma}(z)}{s_{\gamma}(i)}-\frac{s_{\pi / 2}(z)}{s_{\pi / 2}(i)}\right) f(i)+i \pi b \frac{k(i, i)}{s_{\pi / 2}(-i)} \frac{s_{\gamma}(z)}{s_{\gamma}(i)}
\end{aligned}
$$

Note that

$$
\frac{s_{\gamma}(z)}{s_{\gamma}(i)}-\frac{s_{\pi / 2}(z)}{s_{\pi / 2}(i)}=-\frac{\pi \cos \gamma}{s_{\gamma}(i)}(z-i) \frac{k(z,-i)}{s_{\pi / 2}(i)}
$$


so, in view of (26),

$$
\begin{aligned}
\left(\frac{s_{\gamma}(z)}{s_{\gamma}(i)}-\frac{s_{\pi / 2}(z)}{s_{\pi / 2}(i)}\right) f(i) & =\pi b \frac{\tan \gamma+\operatorname{re} \frac{s_{0}(i)}{s_{\pi / 2}(i)}}{\tan \gamma+\frac{s_{0}(i)}{s_{\pi / 2}(i)}}(z-i) \frac{k(z,-i)}{s_{\pi / 2}(i)} \\
& =\pi b(z-i) \frac{k(z,-i)}{s_{\pi / 2}(i)}-i \pi b \frac{\operatorname{im} \frac{s_{0}(i)}{s_{\pi / 2}(i)}}{\tan \gamma+\frac{s_{0}(i)}{s_{\pi / 2}(i)}}(z-i) \frac{k(z,-i)}{s_{\pi / 2}(i)}
\end{aligned}
$$

Also,

$$
i \pi b \frac{k(i, i)}{s_{\pi / 2}(-i)} \frac{s_{\gamma}(z)}{s_{\gamma}(i)}=-i b \frac{s_{\gamma}(z)}{\cos \gamma} \frac{\operatorname{im} \frac{s_{0}(i)}{s_{\pi / 2}(i)}}{\tan \gamma+\frac{s_{0}(i)}{s_{\pi / 2}(i)}} .
$$

Thus, the sum of the last two terms in (31) amounts to

$$
\begin{aligned}
3 \text { rd term }+4 \text { th term } & =\pi b(z-i) \frac{k(z,-i)}{s_{\pi / 2}(i)}-i b \operatorname{im} \frac{s_{0}(i)}{s_{\pi / 2}(i)} s_{\pi / 2}(z) \\
& =b\left(s_{\pi / 2}(z) \operatorname{re} \frac{s_{0}(i)}{s_{\pi / 2}(i)}-s_{0}(z)\right)
\end{aligned}
$$

Additionally, a rather tedious computation shows that the first two terms yield

$$
1 \text { st term }+2 \text { nd term }=-b\left(s_{\pi / 2}(z) \text { re } \frac{s_{0}(i)}{s_{\pi / 2}(i)}-s_{0}(z)\right),
$$

thus completing the proof.

Acknowledgments. Part of this work was done while J. H. T. visited IIMASUNAM (Mexico) in the winter of 2016. He deeply thanks them for their kind hospitality.

\section{References}

[1] S. Albeverio and P. Kurasov. Rank one perturbations, approximations, and selfadjoint extensions. J. Funct. Anal. 148 (1997) 152-169.

[2] S. Albeverio and P. Kurasov. Rank one perturbations of not semibounded operators. Integr. Equ. Oper. Theory 27 (1997) 379-400. 
[3] S. Albeverio and P. Kurasov, Singular perturbations of differential operators. London Mathematical Society Lecture Note Series 271. Cambridge University Press, Cambridge, 2000.

[4] Ju. M. Berezanskiu, Expansions in eigenfunctions of selfadjoint operators. American Mathematical Society, Providence, RI, 1968.

[5] L. de Branges, Some Hilbert spaces of entire functions. Proc. Amer. Math. Soc. 10 (1959) 840-846.

[6] L. de Branges, Some Hilbert spaces of entire functions. Trans. Amer. Math. Soc. 96 (1960) 259-295.

[7] L. de Branges, Hilbert Spaces of Entire Functions. Prentice-Hall, Englewood Cliffs, NJ, 1968.

[8] F. Gesztesy and B. Simon, Rank-one perturbations at infinite coupling. J. Funct. Anal., 128 (1995) 245-252.

[9] S. Hassi, H. Langer, and H.S.V. de Snoo, Selfadjoint extensions for a class of symmetric operators with defect numbers $(1,1)$. 15th OT Conference Proceedings (1995) 123-164.

[10] S. Hassi and H.S.V. de Snoo, One-dimensional graph perturbations of selfadjoint relations. Ann. Acad. Sci. Fenn. Math. 22 (1997) 123-164.

[11] S. Hassi, M. Kaltenbäck, and H.S.V. de Snoo. Triplets of Hilbert spaces and Friedrichs extensions associated with the subclass $\mathbf{N}_{1}$ of Nevanlinna functions. J. Operator Theory, 37 (1997) 155-181.

[12] S. Hassi and H.S.V. de Snoo, Nevanlinna functions, perturbation formulas, and triplets of Hilbert spaces. Math. Nachr. 195 (1998) 115-138.

[13] M. Kaltenbäck and H. Woracek, Pontryagin spaces of entire functions I. Integr. Equ. Oper. Theory 33 (1999) 34-97.

[14] M.G. Krein. On a remarkable class of Hermitian operators. C. R. (Doklady) Acad. Sci. URSS (N. S.) 44 (1944) 175-179.

[15] M.G. Krein. On Hermitian operators whose deficiency indices are 1. C. $R$. (Doklady) Acad. Sci. URSS (N. S.) 43 (1944) 323-326. 
[16] M.G. Krein. On Hermitian operators with deficiency indices equal to one. II. C. R. (Doklady) Acad. Sci. URSS (N. S.) 44 (1944) 131-134.

[17] M. Langer and H. Woracek, A characterization of intermediate Weyl coefficients. Monatsh. Math. 135 (2002) 137-155.

[18] C. Remling. Schrödinger operators and de Branges spaces. J. Funct. Anal., 196 (2002) 323-394.

[19] L.O. Silva, G. Teschl and J.H. Toloza. Singular Schrdinger operators as self-adjoint extensions of N-entire operators. Proc. Amer. Math. Soc. 143 (2015) 2103-2115.

[20] L.O. Silva and J.H. Toloza. The class of $n$-entire operators. J. Phys. A 46 (2013) 025202 (23 pp).

[21] L.O. Silva and J.H. Toloza. A class of $n$-entire Schrödinger operators. Complex Anal. Oper. Theory 8 (2014) 1581-1599.

[22] L.O. Silva and J.H. Toloza. On dB spaces with nondensely defined multiplication operator and the existence of zero-free functions. J. Math. Anal. Appl. 421 (2015) 996-1005.

[23] H. Woracek. Existence of zerofree functions $N$-associated to a de Branges Pontryagin space. Monatsh Math 162 (2011) 453506. 